\title{
Amoxicillin Dosing Regimens for the Treatment of Neonatal Sepsis: Balancing Efficacy and Neurotoxicity
}

\author{
Tamara van Donge $^{a} \quad$ Aline Fuchs $^{\mathrm{a}}{ }^{\mathrm{b}}$ Stéphanie Leroux ${ }^{\mathrm{a}} \quad$ Marc Pfister $^{\mathrm{a}}$ \\ Frédérique Rodieux $^{c}$ Andrew Atkinson ${ }^{a}$ Eric Giannoni ${ }^{d}$ \\ John van den Anker a, e, f Julia Bielickib, g
}

\begin{abstract}
aPaediatric Pharmacology and Pharmacometrics Research, University Children's Hospital Basel, Basel, Switzerland; bMedicines for Malaria Venture, Geneva, Switzerland; 'Division of Clinical Pharmacology and Toxicology, Department of Anaesthesiology, Pharmacology, Intensive care, and Emergency Medicine, Geneva University Hospitals, Geneva, Switzerland; ' ${ }^{\mathrm{C} C l i n i c}$ of Neonatology, Department Mother-Woman-Child, Lausanne University Hospital and University of Lausanne, Lausanne, Switzerland; 'Intensive Care and Department of Surgery, Erasmus Medical Center-Sophia Children's Hospital, Rotterdam, The Netherlands; 'Division of Clinical Pharmacology, Children's National Hospital, Washington, DC, USA; 9Paediatric Infectious Diseases Research Group, Institute for Infection and Immunity, St George's, University of London, London, UK
\end{abstract}

\section{Keywords}

Amoxicillin · Dosing regimen - Neonates - pharmacokinetic/ pharmacodynamic targets - Neurotoxicity · Model-based simulations

\begin{abstract}
Introduction: Large variability in neonatal amoxicillin dosing recommendations may reflect uncertainty about appropriate efficacy and toxicity targets. Objective: The aim of this study was to model efficacious and safe exposure for current neonatal amoxicillin dosing regimens, given a range of assumptions for minimal inhibitory concentration (MIC), targeted \% $\mathrm{fT}>\mathrm{MIC}$, and potential for aminopenicillin-related neurotoxicity. Methods: Individual intravenous amoxicillin exposures based on 6 international and 9 Swiss neonatal dosing recommendations, reflecting the range of current dosing approaches, were assessed by a previously developed population pharmacokinetic model informed by neonatal data from an international cohort. Exposure was simulated by attributing each dosing regimen to each patient cohort. End points of interest were \% $\mathrm{fT}>\mathrm{MIC}$ and potential
\end{abstract}

karger@karger.com www.karger.com/neo

Karger

GOPEN ACCESS
C 2020 The Author(s)

Published by S. Karger AG, Basel

This is an Open Access article licensed under the Creative Commons Attribution-NonCommercial-4.0 International License (CC BY-NC) (http://www.karger.com/Services/OpenAccessLicense), applicable to the online version of the article only. Usage and distribution for commercial purposes requires written permission. neurotoxicity using $C_{\max }>140 \mathrm{mg} / \mathrm{L}$ as threshold. Results: None of the dosing regimens achieved targets of $\geq 100 \% \mathrm{fT}>$ MIC at any of the relevant MICs for a desired probability of target attainment (PTA) of $\geq 90 \%$. All regimens achieved a PTA $\geq 90 \%$ for Streptococcus agalactiae (MIC $0.25 \mathrm{mg} / \mathrm{L}$ ) and Listeria monocytogenes (MIC $1 \mathrm{mg} / \mathrm{L}$ ) when targeting $\leq 70 \% \mathrm{fT}$ $>$ MIC. In contrast, none of the regimens resulted in a PTA $\geq 90 \%$ targeting $\geq 70 \% \mathrm{fT}>$ MIC for enterococci (MIC $4 \mathrm{mg} / \mathrm{L}$ ). The maximum amoxicillin concentration associated with potential neurotoxicity was exceeded using 4 dosing regimens $(100 \mathrm{mg} / \mathrm{kg} \mathrm{q} 12,60 / 30 \mathrm{mg} / \mathrm{kg} \mathrm{q} 12 / 8,50 \mathrm{mg} / \mathrm{kg} \mathrm{q} 12 / 8 / 6$, and $50 \mathrm{mg} / \mathrm{kg} \mathrm{q12/8/4)}$ for $\geq 10 \%$ of neonates. Conclusions: The acceptability of regimens is highly influenced by efficacy and toxicity targets, the selection of which is challenging. Novel randomized trial designs combined with pharmacometric modeling and simulation could assist in selecting optimal dosing regimens in this understudied population.

(c) 2020 The Author(s)

Published by S. Karger AG, Basel

Tamara van Donge and Aline Fuchs contributed equally.
Tamara van Donge

Paediatric Pharmacology \& Pharmacometrics Research

Universitäts-Kinderspital Beider Basel (UKBB), Spitalstrasse 33

CH-4031 Basel (Switzerland)

tamara.vandonge@ukbb.ch 


\section{Introduction}

Neonatal sepsis, including possible serious bacterial infection, is a significant driver of antibiotic use. Globally, around 3 million cases of and up to 600,000 deaths due to neonatal sepsis are estimated to occur every year [1]. A penicillin combined with gentamicin is the first-line treatment for neonatal sepsis recommended by the World Health Organization, and the combination of benzylpenicillin or an aminopenicillin with gentamicin accounts for 29 and $15 \%$ of all regimens used in high-income and low/ middle-income countries, respectively $[2,3]$. In countries such as Switzerland and France, ampicillin is not available and amoxicillin is used instead to treat suspected or proven neonatal sepsis.

Despite the longstanding use of amoxicillin for the treatment of neonates, data supporting optimized dosing are limited. In vitro amoxicillin efficacy is time-dependent and the key pharmacokinetic/pharmacodynamic (PKPD) index is the fraction of time during which the antibiotic concentration remains above the minimal inhibitory concentration (MIC) of the targeted pathogen (\%fT > MIC) [4]. Most studies have not investigated amoxicillin pharmacokinetics (PK) across the entire age and disease spectrum of premature and term infants cared for in neonatal units [4-9]. This limits the identification of optimal dosing approaches in a population that is subject to short-term maturational changes in drug clearance during the postnatal period. Therefore, intravenous amoxicillin dosing recommendations show a large variation with a range from 40 to $120 \mathrm{mg} / \mathrm{kg}$ per day administered in 1, 2, 3, or 4 doses with or without dose stratification according to gestational or post-conceptional age. PK modeling and simulation can support antibiotic dose optimization to identify regimens with suboptimal performance under a range of assumptions $[10,11]$. The main objective of this study was to investigate how the variability of currently used neonatal amoxicillin dosing regimens may impact (i) efficacy, given a range of different equally valid assumptions for MICs, PKPD targets, and (ii) safety, given drug concentration associated with potential neurotoxicity in neonates.

\section{Materials and Methods}

Individual amoxicillin exposures based on international and Swiss neonatal dosing recommendations were assessed by a population PK model, which is informed by data from an international cohort.

\section{Identification of Dosing Recommendations}

Intravenous amoxicillin dosing regimens for the indication of neonatal infection ( $<1$ month after birth) were collected from 9 Swiss level III neonatal intensive care units (NICUs) in 2015. Dosing recommendations were identified from 6 international guidelines, namely, the Swiss Agency for Therapeutic Products 2015 (Swissmedic), the British National Formulary for Children 2015, the Neonatal Formulary 7th edition (NNF7), Frank Shann's Drug Doses 2014 (Shann), The Harriet Lane 2014, and Lexicomp 2016. Dose per administration, dosing interval, total daily dose, and demographics used for dynamic dosing regimens were extracted from each recommendation.

\section{Population of Interest}

Our model population (Table 1) was derived from the clinical data for neonates in the Antibiotic Resistance and Prescribing in European Children (ARPEC) study, an international antibiotic prescribing point prevalence study [12]. To reflect the population managed in neonatal care, neonates and young infants with a postmenstrual age (sum of gestational age [GA] and postnatal age [PNA]) of $\leq 44$ weeks, were considered. We consider this cohort to represent neonates likely to be exposed to amoxicillin and, therefore, to reflect our population of interest (no microbiological data were available). Individual amoxicillin exposure was simulated by attributing each dosing regimen to each cohort patient, that is, for each regimen, each patient was simulated once taking demographic characteristics such as age or weight into account as recommended for the regimen of interest.

\section{Model-Based Simulations of Amoxicillin Exposure}

We systematically identified population PK models available in the literature developed by a nonlinear mixed effect modeling approach, which were applicable to a comparable neonatal population. Amoxicillin exposure was simulated over a timeframe of 7 days in total, for each patient and dosing regimen, following the first intravenous dose to assess amoxicillin exposure at initiation of empiric treatment. Exposure was evaluated after the first dose as adequate early empiric treatment is considered to be crucial for clinical outcomes. It was assumed that PK remains linear across the range of doses simulated. Simulations were performed with NONMEM $^{\circledR}$ (version 7.3.0; ICON Development Solutions, Ellicott City, MD, USA) and R (version 3.5.1; R Development Core Team, Vienna, Austria, http://r-project.org) was used for data analysis and graphics. Nonlinear mixed effect models were utilized to perform simulations of amoxicillin exposure and were characterized in terms of fixed effects and random effects. Fixed effects reflect the population average of the model parameters (e.g., clearance). Random effects relate to the variability effects and allow quantification of inter- and intra-individual variability.

\section{Definition of PKPD Targets}

To evaluate the performance of amoxicillin dosing regimens, we considered a variety of antimicrobial outcome parameters informing targeted PKPD indices. First, MICs were based on breakpoints of microorganisms provided by the European Committee on Antimicrobial Susceptibility Testing (EUCAST) and represent various potential target pathogens for amoxicillin in the neonatal 
Table 1. Demographic characteristics of European neonates and infants in the ARPEC database

\begin{tabular}{|c|c|}
\hline Patients & $N(\%)$ \\
\hline Total, $n(\%)$ & $1,063(100)$ \\
\hline Preterm (<37 weeks gestation), $n(\%)$ & $713(67)$ \\
\hline \multicolumn{2}{|l|}{ Demographics } \\
\hline Male, $N(\%)$ & $597(56)$ \\
\hline Gestational age, median (IQR)/[min-max], weeks & $34(29-39) /[22-42]$ \\
\hline Birth weight, median (IQR)/[min-max], kg & $2.1(1.1-3.2) /[0.4-4.8]$ \\
\hline Age,${ }^{a}$ median $(\mathrm{IQR}) /[\min -\mathrm{max}]$, days & $6(3-16) /[0-60]$ \\
\hline$\leq 7$ days, $N(\%)$ & $580(55)$ \\
\hline$>7$ and $\leq 14$ days, $N(\%)$ & $177(17)$ \\
\hline$>14$ and $\leq 28$ days, $N(\%)$ & $186(28)$ \\
\hline$>28$ and $\leq 60$ days, $N(\%)$ & $120(11)$ \\
\hline Weight, ${ }^{\mathrm{a}}$ median $(\mathrm{IQR}) /[\min -\max ], \mathrm{kg}$ & $2.2(1.2-3.2) /[0.5-4.8]$ \\
\hline $\mathrm{PMA},{ }^{\mathrm{a}}$ median $(\mathrm{IQR}) /[\min -\mathrm{max}]$, weeks & $35.6(31-35.2) /[23.7-44]$ \\
\hline
\end{tabular}

PMA, postmenstrual age; PNA, postnatal age. ${ }^{a}$ PNA at the time of assessment. population [13]. Pathogens such as Streptococcus agalactiae (GBS), Listeria monocytogenes, and enterococci were considered of particular interest because they are important etiologic agents for (early- and late-onset) neonatal sepsis [14-17]. The corresponding target MICs were $0.25,1.0$, and $4.0 \mathrm{mg} / \mathrm{L}$, respectively. In order to provide an extensive profile of the probability of target attainment (PTA) for all potential MICs, we investigated MICs ranging from 0.25 to $8 \mathrm{mg} / \mathrm{L}$.

Second, for the PKPD target of $\% f \mathrm{~T}>\mathrm{MIC}$ which is associated with efficacy, 5 interpretations which are proposed in literature were selected; $30 \% f \mathrm{~T}>\mathrm{MIC}$ based on data suggesting to be sufficient to ensure survival in animal models [18]; 50\% $\mathrm{T}>\mathrm{MIC}$ as this has been reported as being associated with maximal bactericidal effects in such models [19]; 70\% $f \mathrm{~T}>\mathrm{MIC}, 100 \% f \mathrm{~T}>\mathrm{MIC}$, and $100 \% f \mathrm{~T}>4 \times \mathrm{MIC}$ as these PKPD indices are being used in betalactam therapeutic drug monitoring [20]. Third, the adequacy of each dosing regimen in reaching appropriate exposure in the population of interest was determined by computing the PTA for each dosing regimen (combination of MIC and PKPD target). A PTA $\geq 90 \%$, meaning that $90 \%$ of the neonatal population achieved the PKPD target, was considered adequate, although the acceptable level of PTA is currently still under debate, with values ranging from 90 to $99 \%$ [21].

In addition to efficacy targets, we evaluated the maximum exposure expected for each neonate in the model population for the different dosing scenarios. We were interested in the proportion of neonates experiencing a maximal concentration $C_{\max }>140$ $\mathrm{mg} / \mathrm{L}$ at steady state (i.e., exposure at day 7 after 1 week of treatment) as this has been described as representing a potentially neurotoxic aminopenicillin exposure in this population [22].

\section{Results}

\section{Identification of Dosing Recommendations}

Six international guidelines and all surveyed Swiss NICUs provided recommendations for intravenous amoxicillin dosing. Fifteen unique dosing regimens were identified and investigated. Dosing regimens ranged from $10 \mathrm{mg} / \mathrm{kg}$ every $12 \mathrm{~h}$ to $50 \mathrm{mg} / \mathrm{kg}$ every $4 \mathrm{~h}$. Total daily doses of amoxicillin in use in Swiss NICUs (50-200 $\mathrm{mg} / \mathrm{kg} /$ day) were higher than those recommended in international guidelines $(20-200 \mathrm{mg} / \mathrm{kg} /$ day) with one exception (Shann; suggesting a maximum total daily dose of $300 \mathrm{mg} / \mathrm{kg}$ ).

\section{Model-Based Simulations of Amoxicillin}

Two different population PK models were retrieved from the literature $[8,9]$. One model was excluded as it studied exclusively term neonates undergoing therapeutic hypothermia [8]. Therefore, 1 population PK model fulfilled the pre-specified criteria and was selected to simulate neonatal amoxicillin exposure [9]. Data from this analysis originated from a prospective, multicenter, $\mathrm{PK}$ study with a sample size of 187 preterm and term infants [9]. Three covariates for amoxicillin clearance in neonates were identified, namely, weight, GA, and PNA. A 2-compartment model with first-order elimination kinetics was identified to describe PK amoxicillin data accurately [9].

\section{Amoxicillin Exposure under Different Dosing \\ Recommendations}

None of the dosing regimens achieved the PKPD targets of $100 \% f \mathrm{~T}>\mathrm{MIC}$ or $100 \% f \mathrm{~T}>4 \times \mathrm{MIC}$ at any of the relevant MICs based on a desired PTA of $\geq 90 \%$ (Table 2). All dosing regimens achieved $30 \% f \mathrm{~T}>$ MIC with an adequate PTA of $\geq 90 \%$ for all relevant MICs (0.25-4.0 $\mathrm{mg} / \mathrm{L}$ ), with the exception of amoxicillin administered at 10-20 mg/kg every $12 \mathrm{~h}$ (Lexicomp and Harriet Lane). 


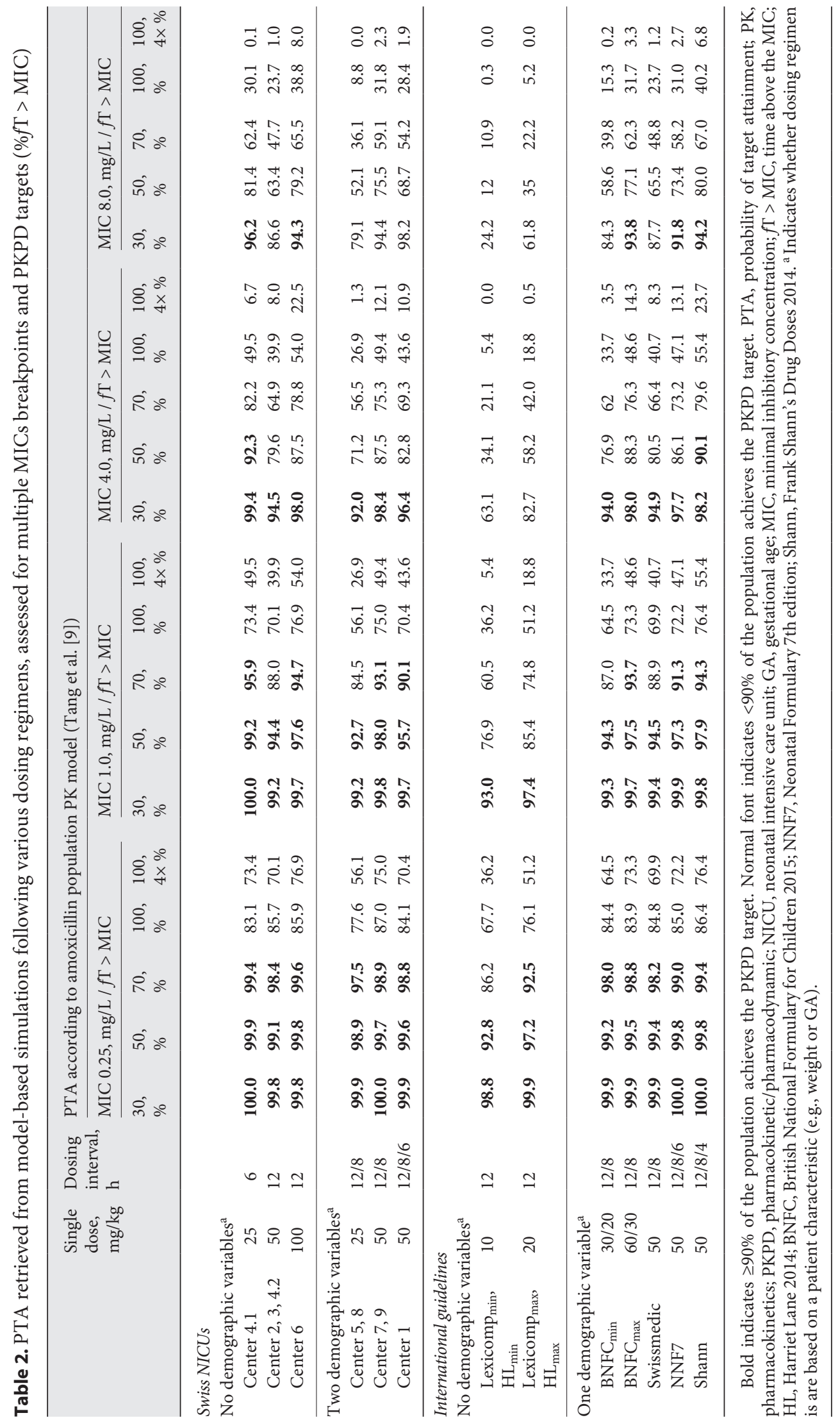




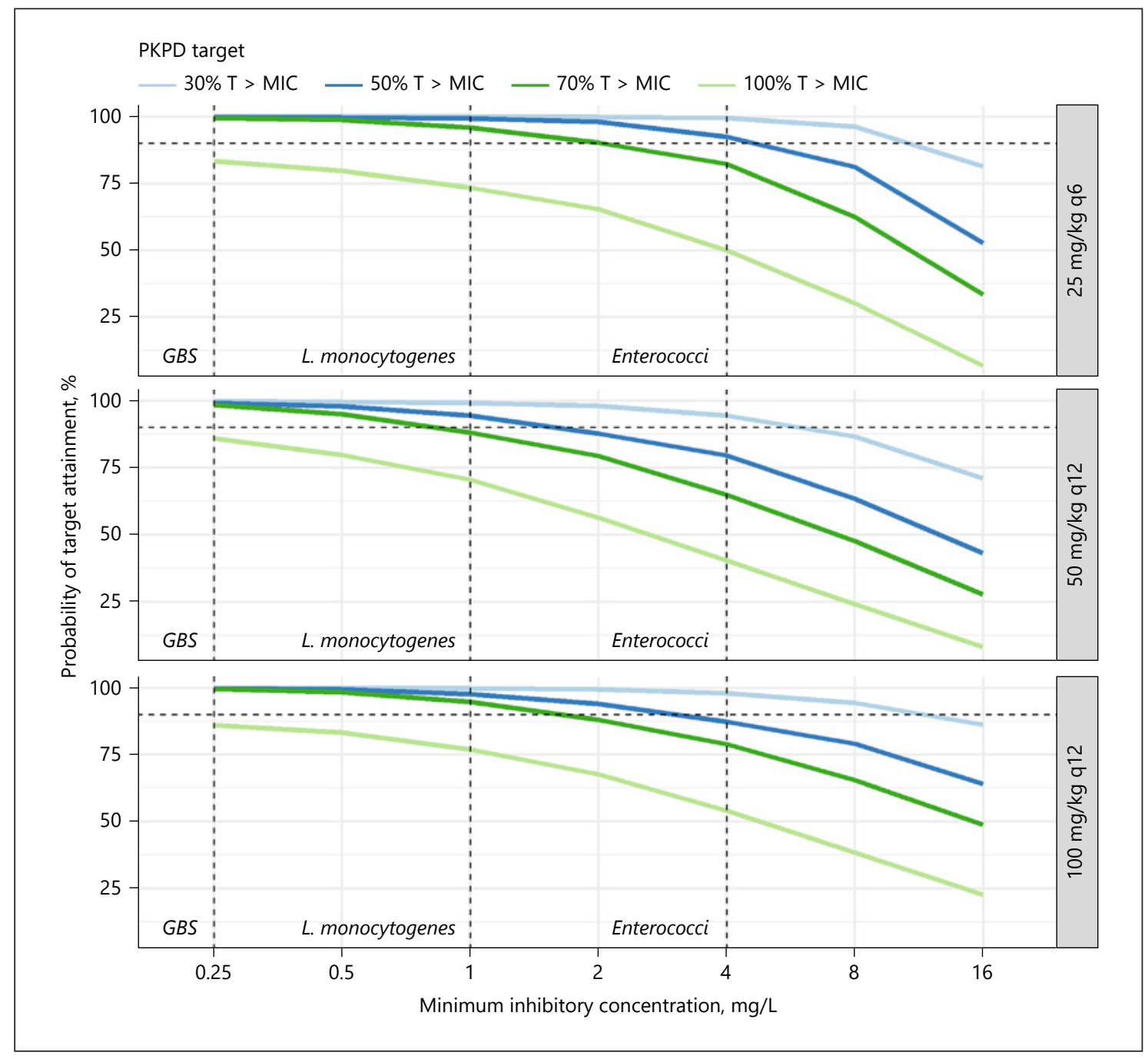

Fig. 1. PTA (\%) retrieved from model-based simulations following various dosing regimens which are not based on any demographic patient characteristics, assessed for multiple MICs breakpoints and PKPD targets (\% $\mathrm{f}$ > MIC). Dotted horizontal line indicates that $\geq 90 \%$ of population achieves PKPD target. PTA, probability of target attainment; GBS, S. agalactiae; MIC, minimal inhibitory concentration; PKPD, pharmacokinetic/pharmacodynamic.

Focusing on targeted MICs of predefined organisms of interest, the majority of regimens achieved a PTA $\geq 90 \%$ for GBS (MIC $0.25 \mathrm{mg} / \mathrm{L}$ ) and L. monocytogenes (MIC 1 $\mathrm{mg} / \mathrm{L}$ ) when targeting $70 \% f \mathrm{~T}>\mathrm{MIC}$ or less, with the exception of $10-20 \mathrm{mg} / \mathrm{kg}$ every $12 \mathrm{~h}$, achieving a PTA $<75 \%$ (Table 2). In contrast, none of the regimens resulted in a PTA $\geq 90 \%$ targeting $70 \% f \mathrm{~T}>$ MIC for enterococci (MIC $4 \mathrm{mg} / \mathrm{L})$. Considering even higher MIC targets, such as the non-species-specific breakpoint of $8 \mathrm{mg} / \mathrm{L}, \mathrm{PTA} \geq 90 \%$ is only achieved for the modest target of $30 \% f \mathrm{~T}>\mathrm{MIC}$ and falls off rapidly for higher PKPD targets (Fig. 1).

Efficacy and Safety of Amoxicillin in Neonates

\section{Amoxicillin Toxicity under Different Dosing Recommendations}

Maximum amoxicillin exposures at steady state when considering different dosing regimens for the model population are shown in Figure 2. For only 4 regimens (100 $\mathrm{mg} / \mathrm{kg}$ every $12 \mathrm{~h}, 60 / 30 \mathrm{mg} / \mathrm{kg}$ every $12 / 8 \mathrm{~h}, 50 \mathrm{mg} / \mathrm{kg}$ every $12 / 8 / 6 \mathrm{~h}$, and $50 \mathrm{mg} / \mathrm{kg}$ every $12 / 8 / 4 \mathrm{~h}$ ) $>10 \%$ of neonates experienced $C_{\max }>140 \mathrm{mg} / \mathrm{L}$. Median $C_{\max }$ for these 4 regimens were 205.7, 123.7, 104.6, and $108.5 \mathrm{mg} / \mathrm{L}$, respectively. Neonates exposed to 3 regimens $(25 \mathrm{mg} / \mathrm{kg}$ every $12 / 8 \mathrm{~h}$ [according to postmenstrual age and PNA], 


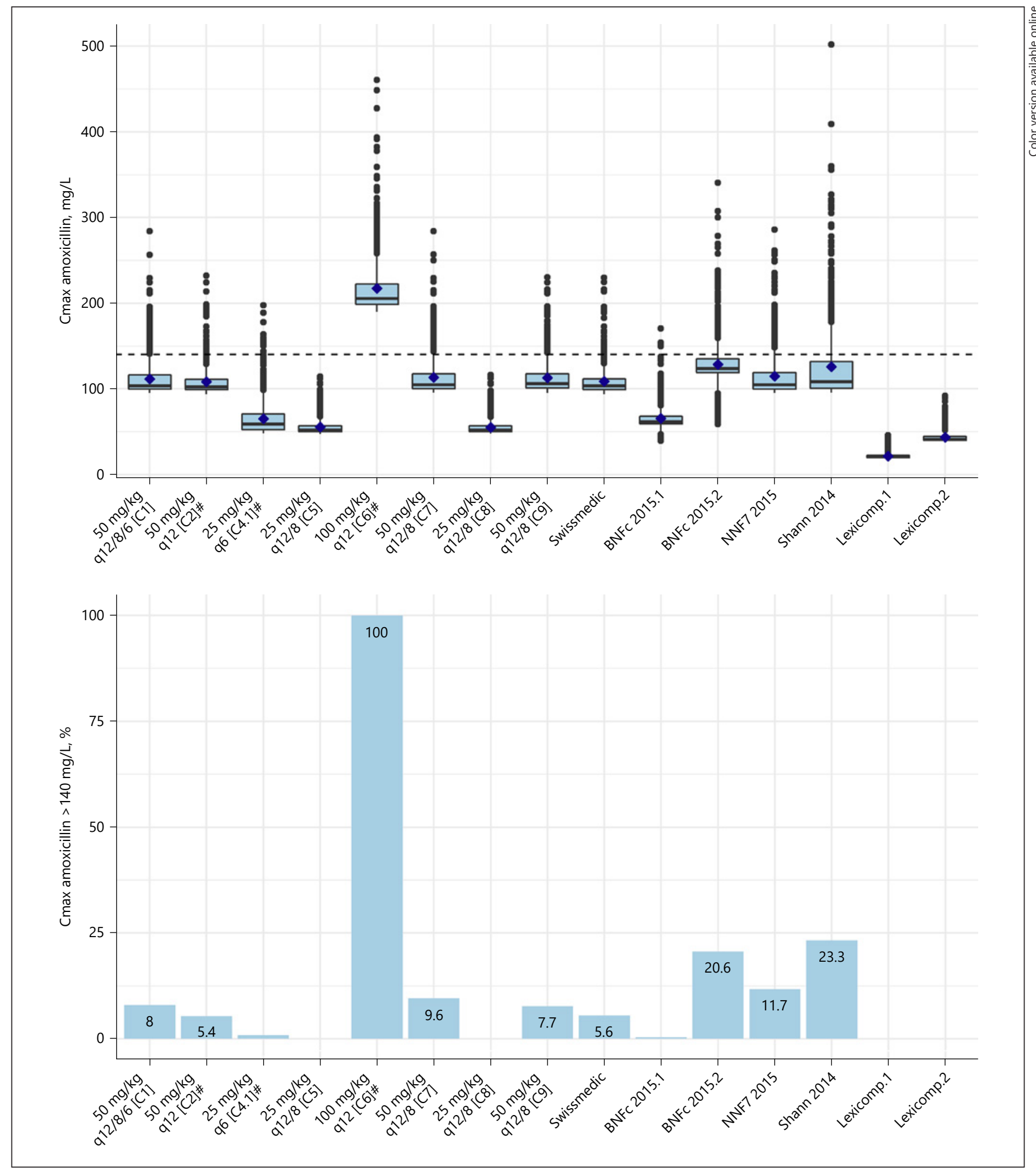

Fig. 2. The maximum amoxicillin concentration levels (upper panel) and the percentage of patients exceeding a maximum amoxicillin concentration of $140 \mathrm{mg} / \mathrm{L}$ (lower panel) according to each of the Swiss national dosing regimens and the international guidelines. Diamond illustrates the mean maximum amoxicillin concentration per center. Dosing regimens discussed in Fig. 1 are highlighted by \#. 
$25 \mathrm{mg} / \mathrm{kg}$ every $12 / 8 \mathrm{~h}$ [according to GA and PNA], and $10 / 20 \mathrm{mg} / \mathrm{kg}$ every $12 \mathrm{~h}$ ) were not expected to experience $C_{\max }>140 \mathrm{mg} / \mathrm{L}$.

\section{Discussion}

Simulating amoxicillin exposure in neonates under various real-life dosing regimens yields 3 key findings. First, at low PKPD targets, $(<70 \% f \mathrm{~T}>\mathrm{MIC})$ and MICs $(\leq 1 \mathrm{mg} / \mathrm{L})$ all regimens perform well, and regimen selection could primarily be driven by avoidance of high and potentially neurotoxic concentrations and by simplicity of dosing regimen for readiness of implementation. Second, at high PKPD targets (70\% $\mathrm{f}$ T $>$ MIC or greater) or MICs $(>2 \mathrm{mg} / \mathrm{L})$, none of the regimens are expected to result in PTA $\geq 90 \%$, indicating that amoxicillin may not be effective enough in such situations. Third, for enterococci (MIC $4 \mathrm{mg} / \mathrm{L}$ ), amoxicillin may be acceptable under certain dosing regimens if the PKPD target is $<70 \% f \mathrm{~T}>$ MIC, but appropriate regimens may result in a large proportion of neonates experiencing high $C_{\max }$ concentrations $>140 \mathrm{mg} / \mathrm{L}$. Our analysis highlights the paradox of identifying optimal amoxicillin dosing regimens in neonates: the acceptability of regimens will differ according to selected PKPD targets (for efficacy and toxicity) and MICs, the selection of which in turn is challenging and highly influenced by microbiological epidemiology of neonatal sepsis (dominant pathogens and their resistance patterns). Rather than specifying dosing regimen based on individual patient characteristics such as weight, PNA, or GA, it could be more appropriate to determine dosing decisions on the exact indication and its microbiological epidemiology: the assumptions (PKPD targets and MIC levels) informed by the target infection contribute to a large extent to target attainment compared to the individual patient characteristics.

As amoxicillin efficacy is time dependent, maximization of amoxicillin concentrations above MIC throughout the dosing interval is theoretically desirable. However, the exact duration of the dosing interval that needs to be targeted remains a matter of debate $[19,20]$. In clinical practice, higher $\% f \mathrm{~T}>\mathrm{MIC}$ are often targeted than indicated by experimental data [20]. This may be especially relevant for preterm neonates given their relative immunological immaturity resulting in altered pharmacodynamics compared with studied adult populations [23]. Data from robust in vivo interventional trials to support such higher targets for improved patient outcomes are lacking. The potential benefit of continuous infusions is being dis-

Efficacy and Safety of Amoxicillin in Neonates cussed for many beta-lactams, especially for critically ill patients [24]. Given the lack of data to support a positive clinical impact of this approach and since the target pathogens with highest MIC for amoxicillin (e.g., enterococci) account for a relatively small proportion of culture-confirmed neonatal sepsis episodes, it remains unclear that routine use of continuous amoxicillin infusion at the empiric treatment stage is justifiable [16]. Practically speaking, intravenous access in this population is often precarious, central venous lines are avoided whenever possible.

Presumably, the rationale for higher PKPD targets used in clinical practice than supported by in vitro data is that beta-lactams are considered extremely safe because of their wide therapeutic window. Data linking high levels of beta-lactam exposure to neurotoxic events in vulnerable populations, however, are accumulating [25-28]. Shaffer and colleagues described ampicillin-induced neurotoxicity in very low birth weight neonates [22]. Of note, the investigation of neurotoxicity in this population is challenging because acute symptoms may remain unnoticed due to their subtle nature. Furthermore, both shortand long-term neurotoxic effects of amoxicillin can be hard to distinguish from effects of the disease itself [25].

We were unable to identify any additional robust data on potential neurotoxic effects of aminopenicillins when administered to neonates, including which parameter is relevant for toxic effects ( $C_{\max }$, area under the curve, and time above concentration). The fact that poor neurodevelopmental outcomes are seen not only in infants with culture-proven sepsis but also in culture-negative infants exposed to antibiotic treatment when compared to unexposed infants underscores the importance of considering the contribution of antibiotics, including commonly used beta-lactams $[29,30]$. We therefore hypothesize that clinically used amoxicillin doses may result in toxicity in some exposed neonates, representing an upper limit of its therapeutic window.

Limitations of this study should be recognized. Regarding empirical treatment when the pathogen is unknown, combination therapy with an aminoglycoside is the first-line treatment of choice with the aim to broaden the coverage of the spectrum of the pathogens. Aminoglycosides target mainly Gram-negative bacteria and staphylococci and are not appropriate for other Grampositive bacteria. Evidence on synergistic antibacterial evidence is lacking, and only in a few situations (e.g., endocarditis) are robust data available to support this effect. Only few population PK models for amoxicillin in preterm and term neonates exist $[6,8,9]$. The model of Tang et al. [9] was preferred since it was developed with pro- 
spectively collected data originating from a multicenter study and extensively validated (internal and external). A large sample size was used $(n=187)$ to develop and validate this model, and the studied population provides an appropriate representation of our target neonatal population. The following covariates were incorporated in the model; GA and PNA as a function of maturation on clearance and weight on volume of distribution and on clearance (by allometric scaling). Additionally, Bayesian TDM tools incorporating post hoc Bayesian estimation based on opportunistic blood sampling and integrating individual targets could further support personalized dosing regimen selection and adjustment.

\section{Conclusion}

At present, simulations may be used only to identify regimens more or less likely to be appropriate in the context of a specific microbiological epidemiology of neonatal sepsis. A small set of potential dosing regimens emerge as unlikely to be suitable even under very conservative assumptions (low targeted $\% f \mathrm{~T}>\mathrm{MIC}$ or low MIC)since acceptable target attainment cannot be expected. The acceptability of all other dosing regimens will strongly be influenced by expected MICs and optimal PKPD targets. The selection of these targets is in turn challenging without clear and robust clinical data linking them to key outcomes. For Switzerland, the presented modeling provided information in selecting a harmonized neonatal intravenous amoxicillin dosing regimen since reliable information on microbiological epidemiology is available. Implementing demographic patient characteristics in the dosing selection process is necessary but not sufficient. Both the impact of development and maturation (reflected by patient characteristics) and microbiological epidemiology (reflected by targeted pathogens or MIC levels) are needed for optimal antimicrobial dosing in neonates. Therefore, better in vivo characterization of PKPD parameters, preferably using novel randomized trial de- signs combined with pharmacometric modeling and simulation, could considerably improve beta-lactam use with respect to efficacy and toxicity in this understudied, vulnerable population.

\section{Acknowledgements}

We thank all physicians and medical personnel from the hospitals that helped us with data acquisition and the following members of the expert committee of the SwissPedDose project: Thomas M. Berger and René Glanzmann. We thank Herman Goossens and Ann Versporten, University of Antwerp and ARPEC project group, for providing access to the ARPEC dataset. We thank Mike Sharland and Angela Huttner for their critical comments that greatly improved the manuscript.

\section{Statement of Ethics}

No ethics approval was required for this study.

\section{Conflict of Interest Statement}

M.P. has parttime employment with the consulting company Certara. Other authors declare no conflict of interest.

\section{Funding Sources}

This study was supported by the Eckenstein-Geigy Foundation and was part of the SwissPedDose project supported by the Swiss Federal Public Health Office. EG is supported by the Leenaards Foundation.

\section{Author Contributions}

T.v.D., A.F., and J.B. designed the study. F.R., A.F., E.G., and M.P. were responsible for data acquisition. T.v.D., A.F., S.L., A.A., and J.B. analyzed and interpreted results. T.v.D., A.F., and J.B. wrote the initial draft. S.L., M.P., J.v.d.A., and J.B. revised the manuscript for important intellectual content. All authors provided significant contributions and approved final version of the submitted article.

\section{References}

1 Fleischmann-Struzek C, Goldfarb DM, Schlattmann P, Schlapbach LJ, Reinhart K, Kissoon N. The global burden of paediatric and neonatal sepsis: a systematic review. Lancet Respir Med. 2018;6(3):223-30.

2 WHO. Pocket book of hospital care for children: guidelines for the management of common childhood illnesses. World Health Organization; 2013.
3 Jackson C, Hsia Y, Basmaci R, Bielicki J, Heath PT, Versporten A, et al. Global divergence from World Health Organization treatment guidelines for neonatal and pediatric sepsis. Pediatr Infect Dis J. 2019;38(11):1104-6.

4 Barker CI, Germovsek E, Hoare RL, Lestner JM, Lewis J, Standing JF. Pharmacokinetic/ pharmacodynamic modelling approaches in paediatric infectious diseases and immunology. Adv Drug Deliv Rev. 2014;73:127-39. 
5 Huisman-de Boer JJ, Van den Anker JN, Vogel M, Goessens WH, Schoemaker RC, de Groot R. Amoxicillin pharmacokinetics in preterm infants with gestational ages of less than 32 weeks. Antimicrob Agents Chemother. 1995;39(2):431-4.

6 Pullen J, Stolk LM, Nieman FH, Degraeuwe PL, van Tiel FH, Zimmermann LJ. Population pharmacokinetics and dosing of amoxicillin in (pre)term neonates. Ther Drug Monit. 2006;28(2):226-31.

7 Pullen J, Driessen M, Stolk LM, Degraeuwe PL, van Tiel FH, Neef C, et al. Amoxicillin pharmacokinetics in (preterm) infants aged 10 to 52 days: effect of postnatal age. Ther Drug Monit. 2007;29(3):376-80.

8 Bijleveld Y, Mathôt R, van der Lee J, Groenendaal F, Dijk P, van Heijst A, et al. Population pharmacokinetics of amoxicillin in term neonates undergoing moderate hypothermia. Clin Pharmacol Ther. 2018;103(3): $458-67$.

9 Tang BH, Wu YE, Kou C, Qi YJ, Qi H, Xu HY, et al. Population pharmacokinetics and dosing optimization of amoxicillin in neonates and young infants. Antimicrob Agents Chemother. 2019;63(2):e02336-18.

10 Sy SK, Zhuang L, Derendorf H. Pharmacokinetics and pharmacodynamics in antibiotic dose optimization. Expert Opin Drug Metab Toxicol. 2016;12(1):93-114.

11 van Donge T, Pfister M, Bielicki J, Csajka C, Rodieux F, van den Anker J, et al. Quantitative analysis of gentamicin exposure in neonates and infants calls into question its current dosing recommendations. Antimicrob Agents Chemother. 2018;62(4):e02004-17.

12 Versporten A, Bielicki J, Drapier N, Sharland M, Goossens H, Group AP, et al. The Worldwide Antibiotic Resistance and Prescribing in European Children (ARPEC) point prevalence survey: developing hospital-quality indicators of antibiotic prescribing for children. J Antimicrob Chemother. 2016;71(4):110617.
13 EUCAST. The European Committee on Antimicrobial Susceptibility Testing. Breakpoint tables for interpretation of MICs and zone diameters. Version 7.1; 2017.

14 Muller-Pebody B, Johnson AP, Heath PT, Gilbert RE, Henderson KL, Sharland M, et al. Empirical treatment of neonatal sepsis: are the current guidelines adequate? Arch Dis Child Fetal Neonatal Ed. 2011;96(1):F4-8.

15 Schrag SJ, Farley MM, Petit S, Reingold A, Weston EJ, Pondo T, et al. Epidemiology of invasive early-onset neonatal sepsis, 2005 to 2014. Pediatrics. 2016;138(6):e20162013.

16 Agyeman PKA, Schlapbach LJ, Giannoni E, Stocker M, Posfay-Barbe KM, Heininger U, et al. Epidemiology of blood culture-proven bacterial sepsis in children in Switzerland: a population-based cohort study. Lancet Child Adolesc Health. 2017;1(2):124-33.

17 Ladhani SN, Henderson KL, Muller-Pebody B, Ramsay ME, Riordan A. Risk of invasive bacterial infections by week of age in infants: prospective national surveillance, England, 2010-2017. Arch Dis Child. 2019;104(9): 874-8.

18 Craig WA. Pharmacokinetic/pharmacodynamic parameters: rationale for antibacterial dosing of mice and men. Clin Infect Dis. 1998 26(1):1-10.

19 Lodise TP, Lomaestro BM, Drusano GL. Application of antimicrobial pharmacodynamic concepts into clinical practice: focus on betalactam antibiotics: insights from the Society of Infectious Diseases Pharmacists. Pharmacotherapy. 2006;26(9):1320-32.

20 Wong G, Sime FB, Lipman J, Roberts JA. How do we use therapeutic drug monitoring to improve outcomes from severe infections in critically ill patients? BMC Infect Dis. 2014;14(1): 288.
21 Mouton JW, Brown DF, Apfalter P, Cantón R, Giske CG, Ivanova M, et al. The role of pharmacokinetics/pharmacodynamics in setting clinical MIC breakpoints: the EUCAST approach. Clin Microbiol Infect. 2012;18(3): E37-45.

22 Shaffer CL, Davey AM, Ransom JL, Brown YL, Gal P. Ampicillin-induced neurotoxicity in very-low-birth-weight neonates. Ann Pharmacother. 1998;32(4):482-4.

23 Strunk T, Richmond P, Simmer K, Currie A, Levy O, Burgner D. Neonatal immune responses to coagulase-negative staphylococci. Curr Opin Infect Dis. 2007;20(4):370-5.

24 Abdul-Aziz MH, Dulhunty JM, Bellomo R, Lipman J, Roberts JA. Continuous beta-lactam infusion in critically ill patients: the clinical evidence. Ann Intensive Care. 2012 Aug 16;2(1):37.

25 Grill MF, Maganti RK. Neurotoxic effects associated with antibiotic use: management considerations. Br J Clin Pharmacol. 2011; 72(3):381-93.

26 Mattappalil A, Mergenhagen KA. Neurotoxicity with antimicrobials in the elderly: a review. Clin Ther. 2014;36(11):1489-511.e4.

27 Payne LE, Gagnon DJ, Riker RR, Seder DB, Glisic EK, Morris JG, et al. Cefepime-induced neurotoxicity: a systematic review. Crit Care. 2017;21(1):276.

28 Quinton MC, Bodeau S, Kontar L, Zerbib Y, Maizel J, Slama M, et al. Neurotoxic concentration of piperacillin during continuous infusion in critically ill patients. Antimicrob Agents Chemother. 2017;61(9):e00654-17.

29 Stoll BJ, Hansen NI, Adams-Chapman I, Fanaroff AA, Hintz SR, Vohr B, et al. Neurodevelopmental and growth impairment among extremely low-birth-weight infants with neonatal infection. JAMA. 2004;292(19):2357-65.

30 Savioli K, Rouse C, Susi A, Gorman G, HisleGorman E. Suspected or known neonatal sepsis and neurodevelopmental delay by 5 years. J Perinatol. 2018;38(11):1573-80. 